\title{
Validity and Reliability of the Spasticity-Associated Arm Pain Scale
}

Klemens Fheodoroff ${ }^{1^{*}}$, Peter Kossmehl ${ }^{2}$ and Jörg Wissel ${ }^{2,3}$

${ }^{1}$ Gailtal-Klinik, Hermagor, Austria

${ }^{2}$ Vivantes Humboldt-Klinikum, Berlin, Germany

${ }^{3}$ Vivantes Klinikum Spandau, Berlin, Germany

*Corresponding author: Klemens Fheodoroff, Gailtal-Klinik, Radniger Strasse 12, A-9620 Hermagor,

Austria, Tel: 4342822220 71101; Fax: 4342822220 70189; E-mail: klemens.fheodoroff@me.com

Received date: July 07, 2017; Accepted date: July 21, 2017; Published date: July 28, 2017

Copyright: (c) 2017 Klemens Fheodoroff, et al. This is an open-access article distributed under the terms of the Creative Commons Attribution License, which permits unrestricted use, distribution, and reproduction in any medium, provided the original author and source are credited.

\section{Abstract}

Objective: Validated, reliable instruments to assess spasticity-related arm pain are not available. Non-specific pain-assessment scales have not been validated in this condition either and may be unsuitable for nursing-home patients. Without such validated scales, the effects of botulinum toxin on this condition cannot be investigated in a scientifically robust manner. The objective of this study was to evaluate the internal consistency, reliability, and validity of the Spasticity-Associated Arm Pain Scale (SAAPS) for adults with post-stroke upper-limb spasticity, and its sensitivity for detecting pain reduction following incobotulinumtoxinA treatment.

Methods: Psychometric evaluation of a five-item pain-assessment tool was conducted in this prospective, multicenter, open-label, observational study, involving adults with post-stroke upper-limb spasticity (inter-rater reliability, $n=25$; all other measures, $n=61$ ). Internal consistency was analyzed using Cronbach's alpha coefficients. Test-retest reliability was assessed using intraclass correlations, Spearman's rho, polychoric correlation, and Kendall's Tau-b coefficients. Inter-rater reliability was assessed using weighted kappa. SAAPS validity was assessed using correlations with patient/investigator ratings on an 11-point numerical rating scale. Sensitivity of SAAPS was investigated 4-6 weeks after an incobotulinumtoxinA injection.

Results: Test-retest reliability was high (all measured coefficients $>0.70$ ) and weighted kappa for inter-rater reliability (0.45-0.69) indicated good/fair agreement. SAAPS scores were reduced by 3.7 points (mean) 4-6 weeks post-treatment $(p<0.0001)$, and indicated pain reduction in $79.7 \%$ of patients. SAAPS scores and numerical rating scale pain ratings were significantly correlated $(p<0.001)$.

Conclusion: SAAPS is a reliable, valid tool for assessing pain reduction after incobotulinumtoxin A treatment in adults with post-stroke upper-limb spasticity.

Keywords Botulinum toxin type A; Geriatric; Muscle spasticity; Nursing home; Observational study; Pain; Validation study

\section{Introduction}

The clinical picture of spasticity in adults following acute brain injury, such as stroke, typically includes velocity-dependent overactivity and muscle stiffness with a tendency for muscle shortening in the muscles involved, loss of fine motor control, muscle spasms, paresis, and changes in limb posture, which may contribute to mobility and self-care limitations, determining and maintaining the need for assistance in these activities [1]. This may negatively impact selfesteem, body image, and mood, and thus diminish quality of life $[2,3]$.

Many patients with spasticity also suffer pain. In a recent cohort study of 974 patients with upper-limb spasticity, musculoskeletal and spasticity-associated pain was reported in up to $50 \%$ of patients, and pain relief was identified as a common goal of treatment [4].

Treatment recommendations for adult spasticity include the administration of botulinum neurotoxin type A (BoNT/A) as part of an integrated treatment program to reduce hypertonia of affected muscles and support intensive physical rehabilitation [1]. A recent evidence-based review of controlled, randomized studies concluded level A evidence for the effectiveness of BoNT/A for upper-limb spasticity in adults [5]. While most clinical studies investigating the effectiveness of BoNT/A have focused on assessing muscle tone, the effect of BoNT/A on spasticity-related pain is less well characterized. A meta-analysis of clinical trials of BoNT/A in adults with spasticity reported that the quality of available evidence for the effectiveness of BoNT/A on spasticity-related pain was poor due to inadequate study designs, highlighting the need for further research with appropriate patient populations and pain assessments [6].

Validated and reliable instruments to assess pain associated with arm spasticity are not yet available. Most studies assessing spasticityrelated pain have used non-specific pain-assessment scales, commonly an 11-point box scale (i.e., a 0-10 numerical rating scale [NRS]) or visual analog scale (VAS). However, such scales have not been validated in patients with spasticity-related arm pain and may lack the sensitivity to detect change [7]. They may also be unsuitable for use by patients in nursing care homes, many of whom have severe cognitive impairment or late-stage dementia [8].

To address these shortcomings, we developed the SpasticityAssociated Arm Pain Scale (SAAPS), a pain-assessment scale for 
routine use in adults with post-stroke arm spasticity that follows the standardized assessment procedure for collecting Ashworth Scale muscle-tone data [9]. The SAAPS collects data on the verbal/ physiological response to passive range of motion (ROM) in five arm segments (Table 1).

A verbal response is denoted by a verbal expression of pain (i.e., "ouch"). A physiological response is denoted by a change in breathing with or without voice (sighing, moaning), a change in visual expression, a tone increase, and/or a withdrawal reaction. Passive movements are performed in a standardized way within the free passive ROM at moderate speed, avoiding undue application of force and below the threshold for eliciting a stretch reflex. Verbal and/or physiological responses are rated on a four-point Likert-type scale as 0 ("no pain"), 1 ("pain on repeated movement" [maximum of five repetitions]), 2 ("pain on end-range movement"), or 3 ("immediate pain on movement"). The SAAPS sum score ranges from 0 to 15 points.

\begin{tabular}{|l|l|}
\hline Item & Description \\
\hline 1 & Paretic shoulder abduction with elbow flexed to $90^{\circ}$ \\
\hline 2 & Paretic shoulder external rotation with elbow flexed to $90^{\circ}$ \\
\hline 3 & Elbow stretching/extension \\
\hline 4 & Wrist stretching \\
\hline 5 & Finger stretching \\
\hline Score & Observation \\
\hline 0 & No pain \\
\hline 1 & Pain on repeated movement (maximum of five repetitions) \\
\hline 2 & Pain on end-range movement \\
\hline 3 & Immediate pain on movement \\
\hline
\end{tabular}

Table 1: The SAAPS passive range of motion items and ratings.

The individual SAAPS items were defined by expert consensus (Klemens Fheodoroff, Jörg Wissel) and cut-offs between items set according to clinical relevance, i.e., in some patients, pain in this segment occurs during repeated movements (score of 1); or in some patients, pain occurs without repetition when reaching the end of ROM in this segment (score of 2). Another cut-off was set between pain at the end of ROM and pain at the start of movement, which would represent the highest level of pain (score of 3). To exclude sensory triggered pain, the occurrence of pain from the start of movement, but not at touch, is rated. The SAAPS ratings are clinically relevant in terms of care/treatment. At pain level 2, care activities could be delivered within the pain-free ROM whereas pain at level 3 would hinder care activities.

Here, we demonstrate the validity and reliability of the SAAPS in adults with post-stroke arm spasticity and show that the SAAPS is sensitive to change following treatment with incobotulinumtoxinA in a predominantly geriatric population.

\section{Methods}

\section{Data collection and study design}

The dataset used to test the internal consistency, test-retest reliability (i.e., reproducibility of the measurements), validity, and sensitivity to change of the SAAPS comprised a group of 61 patients with poststroke spasticity in nursing homes, in whom a decision for focal incobotulinumtoxinA injections within the registered indications (arm spasticity resulting in flexed wrist and clenched fist) had already been made. Assessments were carried out during a prospective, open-label, single-arm, multicenter, observational study in 18 nursing homes in Austria between October 2010 and July 2012. Ethical approval was provided by the ethics committee of the Medical University of Graz, Austria. All participating patients or their legal representatives provided written, informed consent. The study was registered with the Austrian Federal Office for Safety in Health Care [10].

Eligible patients were adult nursing-home residents with disabling arm spasticity (flexed wrist and clenched fist) after a stroke that had occurred $\geq 3$ months prior to baseline assessment who had been prescribed incobotulinumtoxinA, according to the registered Summary of Product Characteristics [11], by a visiting neurologist. Exclusion criteria included neuromuscular disorders, coagulation disorders, previous treatment with BoNT/A or phenol within the previous 4 months, and treatment with intrathecal baclofen within the previous 2 weeks.

Data were obtained during routine visits by neurologists certified in BoNT/A therapy. The study was observational and all treatment decisions, including the decision to prescribe incobotulinumtoxinA, the selection of target muscles for injections, and dosing per muscle, were at the discretion of the attending neurologist. However, the maximum total dose of incobotulinumtoxinA was limited to $400 \mathrm{U}$. Injections were guided using electromyography, electrical stimulation, or ultrasound. Each patient was followed for 6-12 weeks. The observation period included a baseline assessment (Visit 0), an injection visit 2-4 weeks post-baseline (Visit 1), and a follow-up visit 4-6 weeks post-injection (Visit 2). Neurologists recorded their observations on standardized case report forms.

Treatment efficacy was assessed by changes from baseline to the follow-up visit (4-6 weeks post-injection) in the SAAPS score. Overall pain intensity was rated by patients and neurologists on an 11-point NRS from $0=$ "no pain" to $10=$ "worst imaginable pain". Patients were given a standardized instruction ("On a scale from zero to 10 , how severe would you rate your pain?"). The safety of incobotulinumtoxinA was assessed by adverse-event (AE) documentation throughout the observation period.

The inter-rater reliability of the SAAPS was assessed in an independent sample of 25 patients and is presented in the Supplementary Data.

\section{Patients}

The mean age of the patients was 72.8 years (standard deviation $[S D] \pm 12.7)$ and, for the majority of patients $(70.5 \%),>1$ year had elapsed since their stroke (Table 2). All but one patient reported comorbidities (on average four per patient) and were treated with concomitant medication; 42 patients $(68.9 \%)$ received concomitant physio- and/or occupational therapy. Only 11 of 61 patients (18.0\%) could clean the affected hand independently; 36 (59.0\%) had moderate 
Citation: Fheodoroff K, Kossmehl P, Wissel J (2017) Validity and Reliability of the Spasticity-Associated Arm Pain Scale. J Pain Manage Med 3: 127.

Page 3 of 7

to severe difficulties with cleaning the affected hand, and for 14 patients $(23.0 \%)$ this was impossible at baseline due to spastic contracture and pain.

\begin{tabular}{|c|c|}
\hline Characteristics & $n=61$ \\
\hline Age, years; mean (SD) & $72.8(12.7)$ \\
\hline$\geq 65$ years of age, $n(\%)$ & $42(68.9)$ \\
\hline Female, n (\%) & $35(57.4)$ \\
\hline Body mass index, $\mathrm{kg} / \mathrm{m}^{2} ;$ mean (SD) & $23.9(4.5)$ \\
\hline \multicolumn{2}{|l|}{ Duration of upper-limb spasticity, n (\%) } \\
\hline$\leq 1$ year & $18(29.5)$ \\
\hline$>1$ year & $43(70.5)$ \\
\hline \multicolumn{2}{|l|}{ Arm spasticity, a n (\%) } \\
\hline Left & $34(55.7)$ \\
\hline Right & $21(34.4)$ \\
\hline Bilateral & $6(9.8)$ \\
\hline Continuous concomitant non-pharmacologic therapy, b n (\%) & $42(68.9)$ \\
\hline Physiotherapy & $23(37.7)$ \\
\hline Ergotherapy & $15(24.6)$ \\
\hline Speech therapy & $2(3.3)$ \\
\hline Other & $2(3.3)$ \\
\hline
\end{tabular}

Table 2: Baseline characteristics of the observational study population $(n=61)$.

\section{Statistical analyses}

No formal sample size calculation was conducted. Statistical analyses were performed by Dr Robert Heinz and Partner GmbH (Vienna, Austria). Descriptive summary statistics and frequency tables were generated using TESTIMATE V.6.5 (IDV, Gauting/Munich).

AEs were encoded with the Medical Dictionary for Regulatory Activities (version 15.0). Missing data were not imputed (observed case analysis).

\section{Reliability}

SAAPs items were initially screened by assessing internal correlation coefficients. The psychometric analysis was based on ordinal reliability measures, such as the polychoric correlation, Kendall's Tau-b coefficient [12], and ordinal Cronbach's alpha coefficient [13]. Reliability was estimated by internal consistency, test-retest measurement, and inter-rater reliability, based on polychoric correlations [14-16].

Internal consistency (i.e., the extent to which all items within a scale capture the same construct) was quantified using Cronbach's alpha coefficients [13]. The test-retest reliability used average measure intraclass correlations. Kendall's Tau-b coefficient was also used as a distribution-free correlation measure to account for possible skewness in the dataset.

\section{Validity}

The validity of the SAAPS was assessed by testing the correlation of the SAAPS with corresponding patient and investigator pain ratings based on a commonly accepted standard 11-point NRS.

\section{Sensitivity to change}

The significance of changes was tested using Stata 14.0. Most data were recorded on ordinal scales or deviated significantly from normal distributions so the non-parametric Wilcoxon signed rank test was used.

\section{Results}

\section{Internal consistency and reliability of the SAAPS}

\section{Internal consistency}

Cronbach's alpha coefficients of the whole SAAPS were 0.935 at Visit 0 and 0.937 at Visit 1, indicating a high level of internal consistency. Coefficients decreased when items were deleted from the scale, 
Citation: Fheodoroff K, Kossmehl P, Wissel J (2017) Validity and Reliability of the Spasticity-Associated Arm Pain Scale. J Pain Manage Med 3: 127.

Page 4 of 7

meaning that all individual items were well-fitting items (Table 3). Item-test correlation (range 0.782-0.898) showed that each individual item correlated well with the scale as a whole, and the item-rest correlation (range 0.666-0.826) showed that each item correlated well with a scale computed from the other four items (Table 3 ).

\begin{tabular}{|c|c|c|c|}
\hline Item & $\begin{array}{l}\text { Cronbach's alpha coefficient (if } \\
\text { item deleted) }\end{array}$ & Item-test correlation ${ }^{\mathrm{b}}$ & Item-rest correlation ${ }^{b}$ \\
\hline \multicolumn{4}{|l|}{ Individual items } \\
\hline Shoulder abduction with elbow flexed to $90^{\circ}$ & 0.869 & 0.847 & 0.75 \\
\hline Shoulder external rotation with elbow flexed to $90^{\circ}$ & 0.863 & 0.86 & 0.776 \\
\hline Elbow stretching/extension & 0.85 & 0.898 & 0.826 \\
\hline Wrist stretching & 0.881 & 0.805 & 0.689 \\
\hline Finger stretching & 0.887 & 0.782 & 0.666 \\
\hline SAAPS sum score & 0.935 & & \\
\hline
\end{tabular}

Table 3: Internal consistency reliability of the SAAPS ( $n=61)$.

\section{Test-retest reliability}

When the SAAPS was applied twice to the same group of patients in the observational study (at baseline and at the injection visits 2-4 weeks later), intraclass correlations, Spearman's rho coefficient, polychoric correlation, and Kendall's Tau-b coefficient were all $>0.70$ and were all statistically significantly different from zero $(\mathrm{p}<0.0001)$, indicating high test-retest reliability (Table 4 ).

\begin{tabular}{|c|c|c|c|c|}
\hline Item & $\begin{array}{l}\text { Intraclass coefficient } \\
{[95 \% \mathrm{Cl}]}\end{array}$ & $\begin{array}{l}\text { Spearman's rho } \\
\text { coefficient }\end{array}$ & $\begin{array}{l}\text { Polychoric correlation } \\
\text { coefficient }\end{array}$ & $\begin{array}{l}\text { Kendall's } \\
\text { coefficient }\end{array}$ \\
\hline $\begin{array}{l}\text { Shoulder abduction with elbow flexed to } \\
90^{\circ}\end{array}$ & $0.93[0.88,0.96]^{a}$ & 0.86 & 0.92 & 0.78 \\
\hline $\begin{array}{l}\text { Shoulder external rotation with elbow } \\
\text { flexed to } 90^{\circ}\end{array}$ & $0.93[0.89,0.96]^{a}$ & 0.88 & 0.93 & 0.83 \\
\hline Elbow stretching/ extension & $0.95[0.92,0.97]^{a}$ & 0.92 & 0.97 & 0.87 \\
\hline Wrist stretching & $0.94[0.90,0.97]^{a}$ & 0.9 & 0.94 & 0.85 \\
\hline Finger stretching & $0.90[0.84,0.94]^{\mathrm{a}}$ & 0.82 & 0.9 & 0.78 \\
\hline
\end{tabular}

Table 4: Test-retest reliability of the SAAPS $(n=61)$.

\begin{tabular}{|l|l|l|l|l|}
\hline Assessment time point & $\begin{array}{l}\text { Spearman's rho coefficient } \\
\text { (patient) }\end{array}$ & $\begin{array}{l}\text { Spearman's rho coefficient } \\
\text { (investigator) }\end{array}$ & $\begin{array}{l}\text { Pearson product-moment } \\
\text { coefficient (patient) }\end{array}$ & $\begin{array}{l}\text { Pearson } \\
\text { coefficient (investigator) }\end{array}$ \\
\hline Visit 0 & $0.59^{\mathrm{a}}$ & $0.75^{\mathrm{b}}$ & 0.57 & 0.77 \\
\hline Visit 1 & $0.78^{\mathrm{b}}$ & $0.77^{\mathrm{b}}$ & 0.75 & 0.77 \\
\hline Visit 2 & $0.77^{\mathrm{b}}$ & $0.81^{\mathrm{b}}$ & 0.61 & 0.8 \\
\hline
\end{tabular}

${ }^{a} p<0.001,{ }^{b} p=0.0001$ (Wilcoxon signed rank test),NRS patient ratings were available for 30,32 , and 26 patients at Visits 0 , 1, and 2, respectively. NRS investigator ratings were available for 44,45 , and 45 patients at Visits 0,1 , and 2, respectively.NRS: numerical rating scale; SAAPS: Spasticity-Associated Arm Pain Scale.

Table 5: Validity of the SAAPS ( $\mathrm{n}=61)$ vs. 11-point NRS of overall pain intensity (patient- and investigator-rated). 
Citation: Fheodoroff K, Kossmehl P, Wissel J (2017) Validity and Reliability of the Spasticity-Associated Arm Pain Scale. J Pain Manage Med 3: 127.

Page 5 of 7

\section{Validity of the SAAPS}

Spearman's rho coefficient for the correlation of the SAAPS scores at Visits 0,1 , and 2 with both the patient's and investigator's ratings on a standard 11-point NRS ranged from 0.59-0.81, and the Pearson product-moment coefficients ranged from 0.57-0.80 (Table 5).

\section{Inter-rater reliability}

To test the inter-rater reliability of the SAAPS, a cohort of 25 patients (separate from this study) was tested independently by two investigators.

Near observed agreement (i.e., ratings that do not differ by $>1$ point) was $\geq 80 \%$ (established acceptable level) for all items and absolute observed agreement (i.e., identical ratings) was $\geq 60 \%$ (established acceptable level) for Items 3 and 5 (elbow and finger stretching). (Supplementary Figure S1 and Table S1). Further details are presented in the Supplementary Data (Table S2).

\section{Sensitivity to change after incobotulinumtoxinA treatment}

\section{Treatment}

All 61 patients received a single treatment with incobotulinumtoxinA (mean [SD] 194.8 [88.1] U; range 30-400 U) on the second visit; follow-up data were available for 59 patients.

Injections were at the discretion of the treating neurologist and were performed according to the registered Summary of Product Characteristics [11].

\section{SAAPS assessment of upper-limb pain}

At the follow-up visit 4-6 weeks post-injection with incobotulinumtoxinA, the SAAPS sum score, collected in 59 of 61 patients, was reduced from a mean (SD) baseline score of 7.5 (5.1) to a mean (SD) follow-up score of 3.8 (4.1) $(\mathrm{p}<0.0001$; Figure 1A).

Pain reduction (i.e., a decrease in the SAAPS score) was documented for 47 of 59 patients (79.7\%; Figure 1B). Eleven patients $(18.6 \%)$ experienced no change in pain, and pain increased for one patient.

\section{Overall pain intensity on the 11-point NRS}

Overall pain intensity, rated by the treating neurologist on the 11point NRS, improved from a mean (SD) of $5.3(2.9)$ at baseline $(n=44)$ to $2.6 \quad(2.5)$ at $4-6$ weeks post-injection $(n=45)$ with incobotulinumtoxinA ( $<<0.0001$; Figure 1C).

Investigator-rated pain reduction (i.e., a decrease in NRS score) was reported for 30 of 37 patients (81.1\%; Figure 1B). Six patients (16.2\%) experienced no change and one patient experienced increased pain. Patient ratings of overall pain intensity improved from a mean (SD) of 4.1 (3.0) at baseline $(\mathrm{n}=30)$ to a mean (SD) of $2.6(3.3)$ at follow-up 4-6 weeks post-injection $(n=26 ; p=0.0003$; Figure $1 C)$.

\section{Tolerability of incobotulinumtoxinA}

Two of the 61 patients who received incobotulinumtoxinA treatment (3.3\%) experienced an AE during the observation period. A 78-year-old patient had a mild urinary tract infection considered not related to incobotulinumtoxin $\mathrm{A}$ treatment.
The second AE was a transient ischemic attack of severe intensity in an 80 -year-old patient which was also considered unlikely to be related to the study medication.

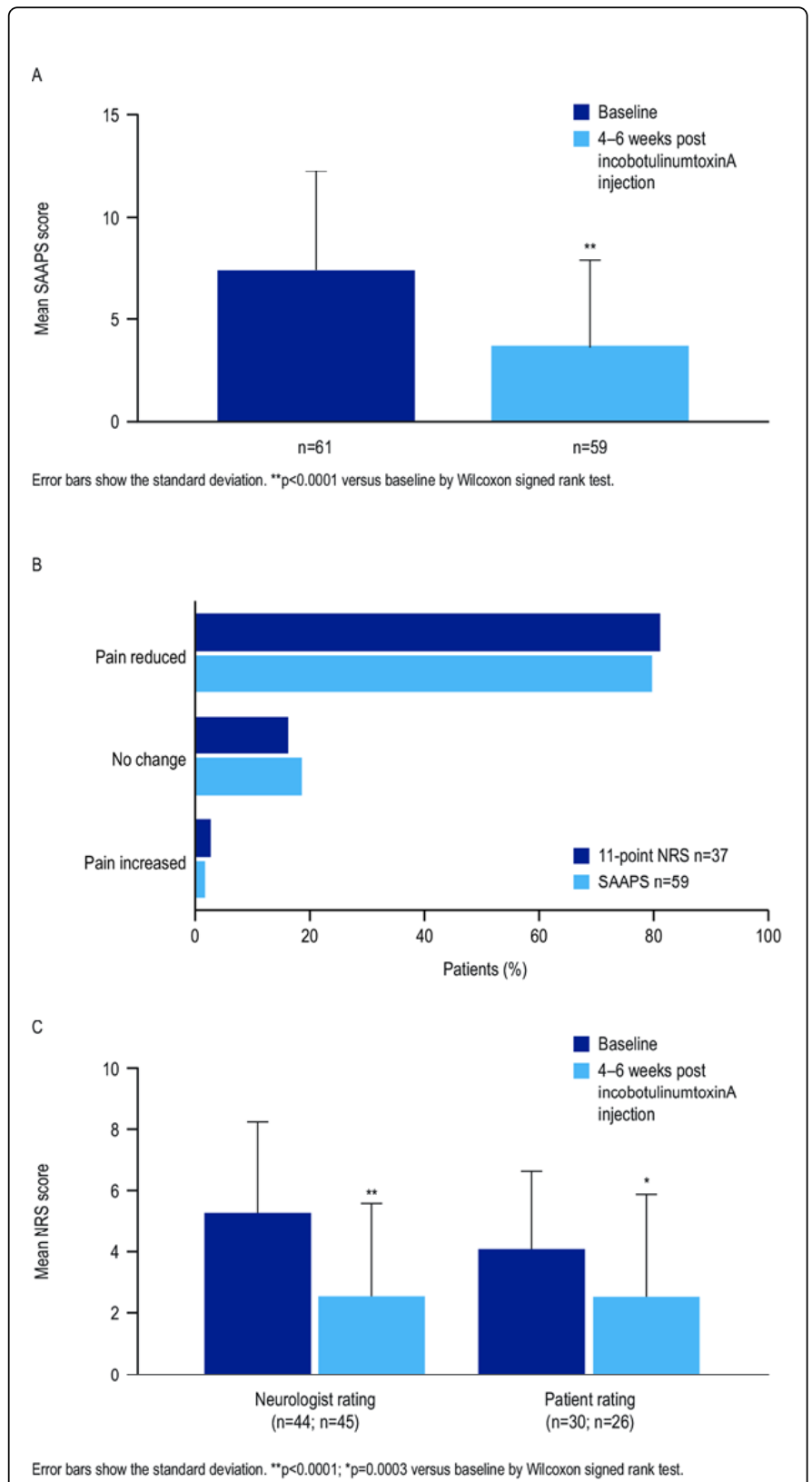

Figure 1: Effect of incobotulinumtoxinA treatment on (A) mean SAAPS score, (B) change in investigator-rated pain intensity, and (C) mean overall pain intensity (11-point NRS). n: number of patients with available data; NRS: numerical rating scale; SAAPS: Spasticity-Associated Arm Pain Scale.

\section{Discussion}

The SAAPS is a new instrument that has been developed specifically for routine assessment of observed arm pain in adult patients with chronic spasticity in the upper limb following stroke. SAAPs was developed following a standardized assessment procedure for 
collecting Ashworth Scale muscle-tone data [9] and the items selected for inclusion were defined by expert consensus. As we were unable to identify any published reference on how to collect data on spasticityassociated arm pain, we decided to assess and report the psychometric properties of the chosen items. Our data demonstrate the internal consistency of the five individual SAAPS items with the scale as a whole, as well as the test-retest and inter-rater reliability of the SAAPS. We have also confirmed the validity of the SAAPS by the correlation of the SAAPS scores with pain ratings captured on the commonly accepted "gold-standard" 11-point NRS. In an observational study of a predominantly geriatric, multi-morbid population of patients, the SAAPS detected reductions in pain after treatment with incobotulinumtoxinA, confirming that the SAAPS was sensitive to change. Moreover, the SAAPS scores were collected in 59 of 61 patients at follow-up, whereas the standard physician-rated 11-point NRS were collected in 45 of 61 patients at follow-up. While the higher rate of collection of the SAAPS may reflect the study focus, the collection of NRS data was also part of the study design. Therefore, this finding suggests that the SAAPS is more convenient to apply than the standard 11-point NRS.

Most previous randomized, controlled clinical trials of BoNT/A in spasticity have focused on efficacy outcomes, assessing the degree of muscle tone or motor symptoms [6]. There is some existing evidence that BoNT/A treatment can also alleviate spasticity-related pain in adults [17-20]. However, a systematic review and meta-analysis of randomized, placebo-controlled clinical trials of BoNT/A in spasticity concluded that the quality of evidence for effects on pain was low or very low and that no significant effect had been demonstrated [6]. This was considered to be largely due to inadequacies in the design of the studies, such as omission of pain from the patient inclusion criteria, and inconsistent use and analysis of pain rating scales. A recent study by Lam et al found that BoNT/A treatment could decrease the caregiver burden for patients with upper-limb spasticity in long-term care, but found no significant effect on pain [21]. In this study, pain was assessed using the Pain Assessment in Advanced Dementia (PAINAD) scale, which was developed for patients with advanced dementia [22]. The scale has been developed for male patients only and has not been validated for the assessment of spasticity-related pain. In addition to PAINAD, several other tools for the assessment of pain in this population have been developed. However, there is a lack of comprehensive evidence on the reliability, validity, and feasibility of their use in clinical practice [8].

\section{Strengths and limitations}

Using the SAAPS allowed us to detect significant effects of BoNT/A treatment on spasticity-associated arm pain in a geriatric population. Our analyses show that all items of the SAAPS are relevant for spasticity-associated pain. Therefore, the SAAPS may provide a more objective measure in these patients than scales that are not diseasespecific such as a NRS or VAS. In addition, the reliability, and validity of most other scales used to assess pain in neurological conditions have not been fully evaluated in patients after stroke [7]. The treating neurologists in our observational study found that the SAAPS required little time to administer and was a useful tool for setting and evaluating treatment goals (personal communication). This suggests that the SAAPS may be feasible for use in standard neurological practice to evaluate spasticity-associated pain after a stroke, including in geriatric patients.
In the absence of any scales that are validated specifically for spasticity-related pain, there were no alternatives to using VAS/NRS pain scales for validation of the SAAPS, although we appreciate such an approach may be considered somewhat cumbersome. A limitation of the SAAPS itself is that the tool has demonstrated evidence of validity in adult patients with chronic arm spasticity after a stroke, but not yet in patients with spasticity of other etiologies. Furthermore, assessment of the SAAPS requires at least some clinical experience in investigating the musculoskeletal system. Severe flaccid subluxation of the shoulder as well as severe hand edema might lead to false-positive pain scores and therefore should be disregarded.

\section{Conclusion}

In conclusion, the SAAPS is a useful tool to assess spasticityassociated arm pain and to document pain reduction after BoNT/A treatment in patients with chronic arm spasticity following stroke, including geriatric patients who frequently have comorbidities such as dementia.

\section{Acknowledgments}

The authors would like to thank Dr Ronald Saurugg, Dr Eva Kronberger, Dr Helmut Grantl-Pühringer, Dr Cornelia Adelwöhrer, Dr Josef Grossmann, Dr Stefan Höger, and Dr Thomas Sycha for the collection of data in the non-interventional study. Editorial assistance with the development of this manuscript was provided by Dr Simone Boldt of Complete Medical Communications and financed by Merz Pharmaceuticals, GmbH.

\section{References}

1. Wissel J, Ward AB, Erztgaard P, Bensmail D, Hecht MJ, et al. (2009) European consensus table on the use of botulinum toxin type A in adult spasticity. J Rehabil Med 41: 13-25.

2. Sturm JW, Donnan GA, Dewey HM, Macdonell RA, Gilligan AK, et al. (2004) Quality of life after stroke: the North East Melbourne Stroke Incidence Study (NEMESIS). Stroke 35: 2340-2345.

3. Zorowitz RD, Gillard PJ, Brainin M (2013) Poststroke spasticity: Sequelae and burden on stroke survivors and caregivers. Neurology 80: S45-S52.

4. Bakheit AM, Zakine B, Maisonobe P, Aymard C, Fhedoroff K, et al. (2010) The profile of patients and current practice of treatment of upper limb muscle spasticity with botulinum toxin type A: An international survey. Int J Rehabil Res 33: 199-204.

5. Esquenazi A, Albanese A, Chancellor MB, Elovic E, Segal KR, et al. (2013) Evidence-based review and assessment of botulinum neurotoxin for the treatment of adult spasticity in the upper motor neuron syndrome. Toxicon 67: 115-128.

6. Baker JA, Pereira G (2013) The efficacy of Botulinum Toxin A for spasticity and pain in adults: A systematic review and meta-analysis using the Grades of Recommendation, Assessment, Development and Evaluation approach. Clin Rehabil 27: 1084-1096.

7. Tyson SF, Brown P (2014) How to measure pain in neurological conditions? A systematic review of psychometric properties and clinical utility of measurement tools. Clin Rehabil 28: 669-686.

8. Lichtner V, Dowding D, Esterhuizen P, Closs SJ, Long AF, et al. (2014) Pain assessment for people with dementia: a systematic review of systematic reviews of pain assessment tools. BMC Geriatr 14: 138.

9. Ashworth B (1964) Preliminary trial of carisoprodol in multiple sclerosis. Practitioner 192: 540-542.

10. https://forms.ages.at/nis/registerDetail.do?id=TklTMDAxMzgz

11. https://www.medicines.org.uk/emc/medicine/20666 
Citation: Fheodoroff K, Kossmehl P, Wissel J (2017) Validity and Reliability of the Spasticity-Associated Arm Pain Scale. J Pain Manage Med 3: 127.

Page 7 of 7

12. Kendall MG (1938) A new measure of rank correlation. Biometrika 30: 81-93.

13. Cronbach L (1951) Coefficient alpha and the internal structure of tests Psychometrika 16: 297-334.

14. Flora DB, Curran PJ (2004) An empirical evaluation of alternative methods of estimation for confirmatory factor analysis with ordinal data. Psychol Methods 9: 466-491.

15. Gadermann AM, Guhn M, Zumbo BD (2012) Estimating ordinal reliability for Likert-type and ordinal item response data: A conceptual empirical, and practical guide. Pract Assess Res Eval 17: 1-13.

16. Zumbo BD, Gadermann AM, Zeisser C (2007) Ordinal versions of coefficients alpha and theta for Likert rating scales. J Mod Appl Stat Methods 6: 21-29.

17. Esquenazi A, Mayer N, Lee S, Brashear A, Elovic E, et al. (2012) Patient registry of outcomes in spasticity care. Am J Phys Med Rehabil 91: 729-746.

18. Shaw LC, Price CI, van Wijck FM, Shackley P, Steen N, et al. (2011) Botulinum Toxin for the Upper Limb after Stroke (BoTULS) Trial: Effect on impairment, activity limitation, and pain. Stroke 42: 1371-1379.
19. Wissel J, Müller J, Dressnandt J, Heinen F, Naumann M, et al. (2000) Management of spasticity associated pain with botulinum toxin A. J Pain Symptom Manage 20: 44-49.

20. Wissel J, Ganapathy V, Ward AB, Borg J, Ertzgaard P, et al. (2016) OnabotulinumtoxinA improves pain in patients with post-stroke spasticity: Findings from a randomized, double-blind, placebo-controlled trial. J Pain Symptom Manage 52: 17-26.

21. Lam K, Lau KK, So KK, Tam CK, Wu YM, et al. (2012) Can botulinum toxin decrease carer burden in long term care residents with upper limb spasticity? A randomized controlled study. J Am Med Dir Assoc 13: 477-484.

22. Warden V, Hurley AC, Volicer L (2003) Development and psychometric evaluation of the Pain Assessment in Advanced Dementia (PAINAD) scale. J Am Med Dir Assoc 4: 9-15. 